\title{
Smart Agriculture with an Automated IoT-Based Greenhouse System for Local Communities
}

\author{
Nianpu Li1 ${ }^{*}$, Yimeng Xiao², Lei Shen ${ }^{3}$, Zhuoyue $\mathrm{Xu}^{4}$, Botao $\mathrm{Li}^{5}$, Chongxuan $\mathrm{Yin}^{6}$ \\ ${ }^{1}$ Beijing No. 80 High School, Beijing, China \\ ${ }^{2}$ Tianjin Polytechnic University, Tianjin, China \\ ${ }^{3}$ Purdue University, West Lafayette, USA \\ ${ }^{4}$ Pace University, New York, USA \\ ${ }^{5}$ Boston University, Boston, USA \\ ${ }^{6}$ The State University of New York at Stony Brook, Long Island, USA \\ Email: ^nian_pu@126.com
}

How to cite this paper: Li, N.P., Xiao, Y.M., Shen, L., Xu, Z.Y., Li, B.T. and Yin, C.X. (2019) Smart Agriculture with an Automated IoT-Based Greenhouse System for Local Communities. Advances in Internet of Things, 9, 15-31.

https://doi.org/10.4236/ait.2019.92002

Received: March 6, 2019

Accepted: April 26, 2019

Published: April 29, 2019

Copyright (c) 2019 by author(s) and Scientific Research Publishing Inc. This work is licensed under the Creative Commons Attribution-NonCommercial International License (CC BY-NC 4.0).

http://creativecommons.org/licenses/by-nc/4.0/

\begin{abstract}
Nowadays, smart agriculture using wireless communication is replacing the wired system which was difficult to install and manage. Then, this paper introduces a new design for IoT application on the greenhouse, which utilizes different technologies to present a new model for practical implementation in the IoT concept. This design can settle a new method to solve problems in Market Demand, Precision in Operation and supervision. Furthermore, this design can be used in many cases and assist farmers, cropper and planted people to develop their business.
\end{abstract}

\section{Keywords}

Internet of Things, Hydroponics, ZigBee, Sensor Network, Automated Agriculture, Cloud

\section{Introduction}

\subsection{Background}

The term Internet of Things, or IoT, was first mentioned by Kevin Ashton in 1999, in his effort to explain how radio frequency identification (RFID) could be incorporated into the Internet. According to the International Telecommunication Union (ITU), the Internet of Things has been defined as "a global infrastructure for the information society, enabling advanced services by interconnecting (physical and virtual) things based on existing and evolving interopera- 
ble information and communication technologies" [1]. The radical evolution of the current Internet in to connect everything that not only converges the information from the environment and cooperate with physical world like actuation, command, and control, but also using the Internet to provide the services for information, transfer, analytics, applications, and communication [2]. Along with the trend of Big Data, Cloud Computing, and Machine Learning, an IoT system integrated with the latest technologies in data processing will potentially solve obstacles and bring advancements to various domains, such as manufacturing, healthcare, logistics, and agriculture. In recent years, governments throughout the world have greatly supported the research and application of IoT in agriculture. One of the models of agriculture combining with IoT is a hydroponic system which demands many devices been connected. As for communication between devices, IEEE 805.15.4 is always chosen as physical layer and access protocol to achieve mesh network communication. IEEE805.15.4, included in IEEE802.15, is a standard of the wireless personal area networks (WPANs) and it is a standard to meet the requirement of low power consumption and low-cost wireless network.

\subsection{Objective}

In this paper, our objective is to develop a greenhouse with an IoT system to serve as a one-stop shop to supply local communities' demands to production. The IoT system helps to achieve the purpose in the following ways:

\subsubsection{Market Demand}

To provide plants for households, restaurants, and grocery stores in the local communities, the IoT system uses machine learning algorithms to analyze historical data of different kinds of crops price, and then predict the quantity demanded and price demanded of particular types of plants in the upcoming local market. Based on the predicted demand of the local market, the IoT system can offer optimal farming condition, produce recommendation of the floristics for users.

\subsubsection{Precision in Operation}

With a wide range of techniques, the IoT system employs automation to control growing conditions in the hydroponic greenhouse. Together, the automated heating/cooling system, the $\mathrm{CO}_{2}$ emissions control system, the LED lights, the nutrient supplement system delivers a unique growing recipe to each plant.

\subsubsection{Supervision}

The IoT system constantly monitors the greenhouse from the data collected by a variety of sensors. If unusual changes take place in the plants or the devices, the IoT system immediately sends alerts to the users via the mobile application. Once emergencies are notified, users are able to access detailed reports.

Comparing to traditional greenhouses, the IoT-based hydroponic greenhouse grows a diversity of plants at the same time to fulfill the basic needs of a specific local community (see Figure 1), which are forecasted by the cloud's analyzing 


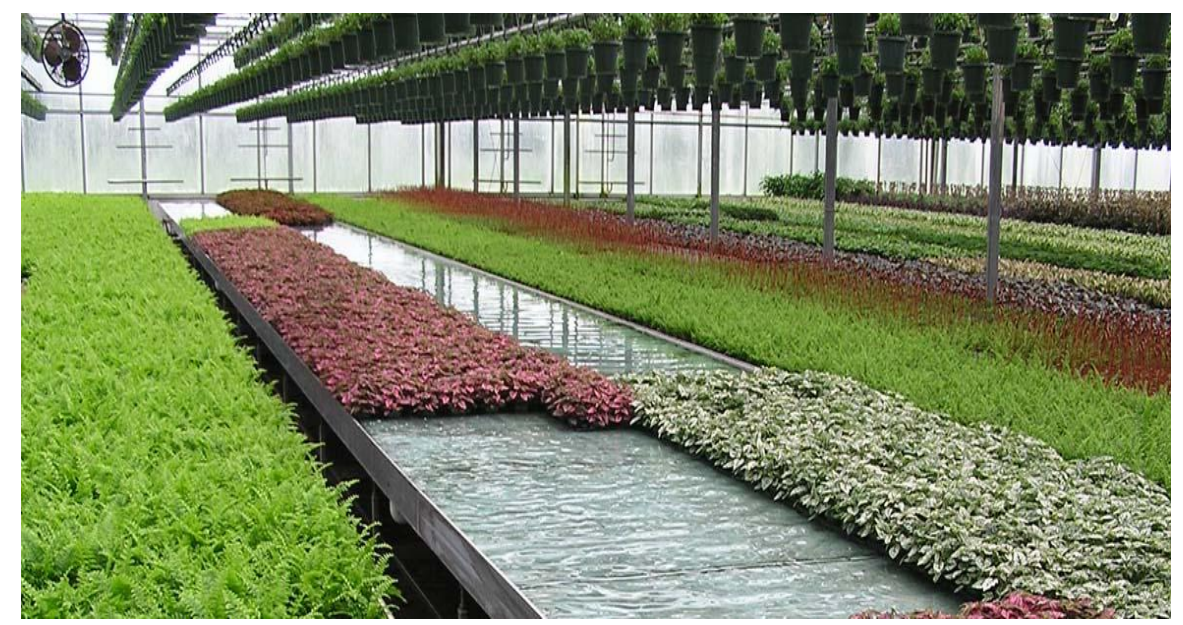

Figure 1. Diversity in greenhouse.

of historical market data. Most importantly, the IoT system uses machines' decision-making and precise actuating to replace human labor in greenhouse production.

\section{The Architecture of the Greenhouse System}

Concerning the integrated system logically, we will set varies of sensors in the greenhouse for collecting data such as $\mathrm{CO}_{2}$ concentration, humidity, illumination intensity and so on. At the same time, there are air pumps, micro-CHP, and other devices which will be controlled by the data sensor collected to keep the environment stable.

As to the data are sent through ZigBee to the local host to update to the cloud which stores the data and where the data is analyzed using linear regression. Beyond that, users could access the cloud to check the plant and get a variety of cognitive recommendations about future marketing decisions. The conspectus of system's workflow can be recognized in Figure 2.

\subsection{System Description}

Given a space about $10 \mathrm{~m}^{2}$, an automated hydroponic greenhouse could be set up with several water tanks with consisting of 3 to 5 containers to grow crops as Figure 3 shown. At the top of a tank is a floating sheet. It can help containers to float above water. At the bottom of the container are the water pump and oxygenator. A tank can have multiple layers as shown in Figure 4.

What's more, we need to control the environment for the greenhouse. There will be 7 factors to control: Humidity, temperature, $\mathrm{CO}_{2}$, light, $\mathrm{EC}$, $\mathrm{pH}$, and dissolved oxygen. Those factors have corresponding sensors and actuators. Sensors will help IoT system to collect these data, and actuators will receive data from cloud to react to corresponding changes on these 7 factors. Data communication between sensors is called a wireless sensor network, which will form a mesh network. After data is being collected, they will be sent to a local server through the sensor network. And the local server will send data to the cloud server by 


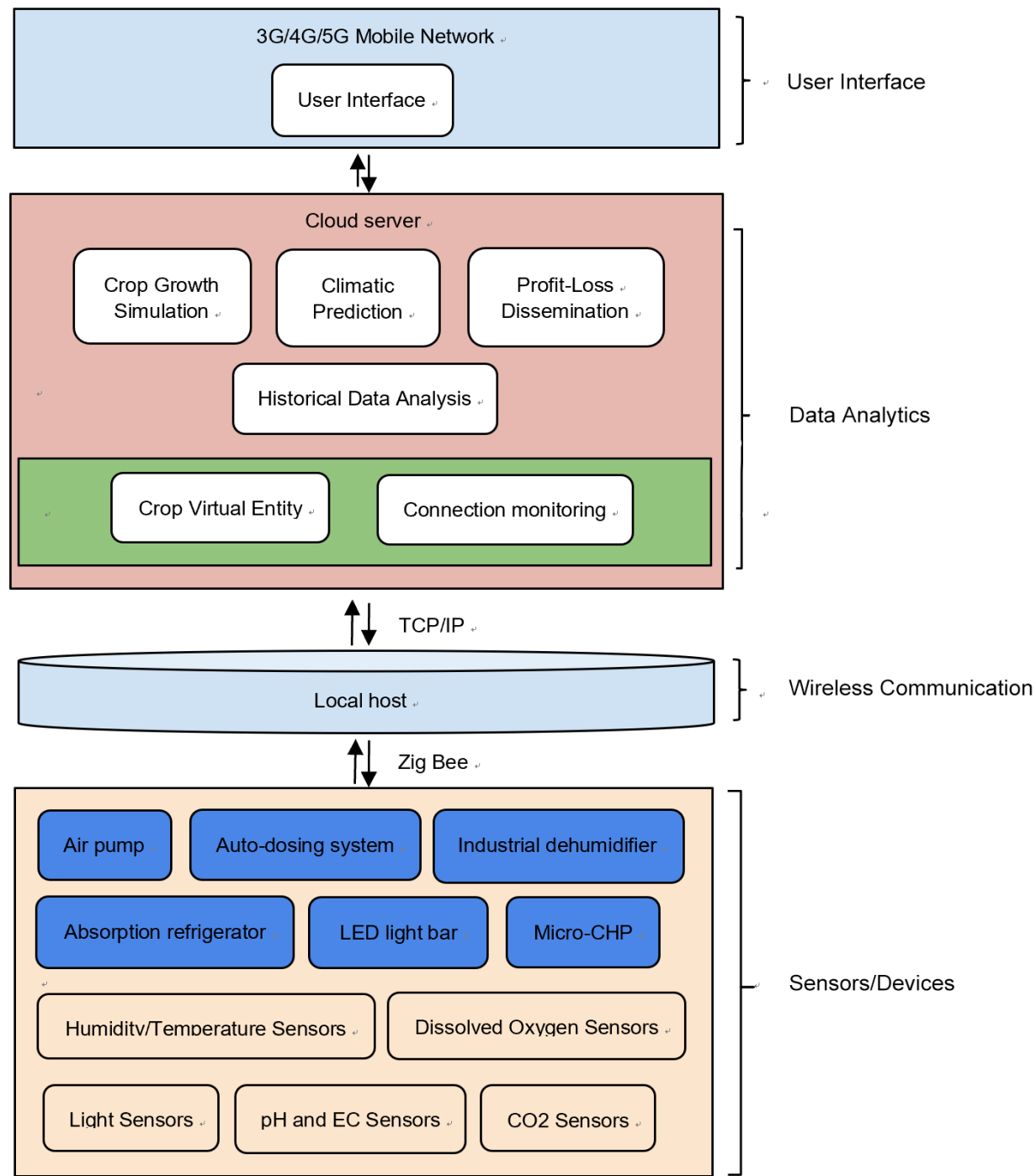

Figure 2. The system layout.

\section{Bubbleponics}

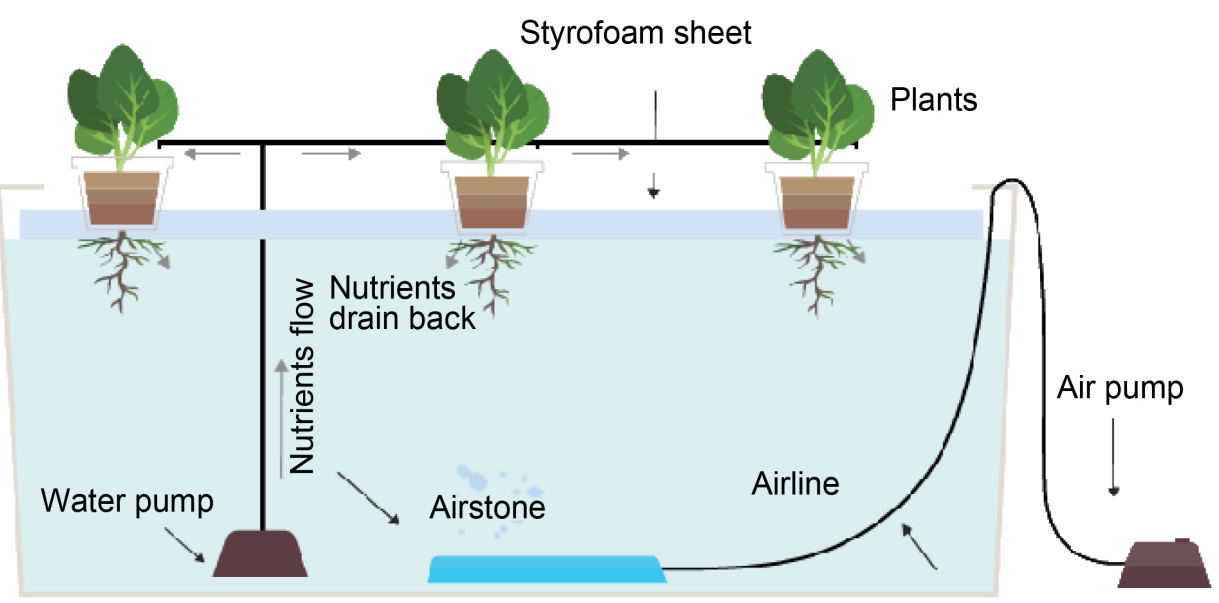

Figure 3. Bubbleponics. 


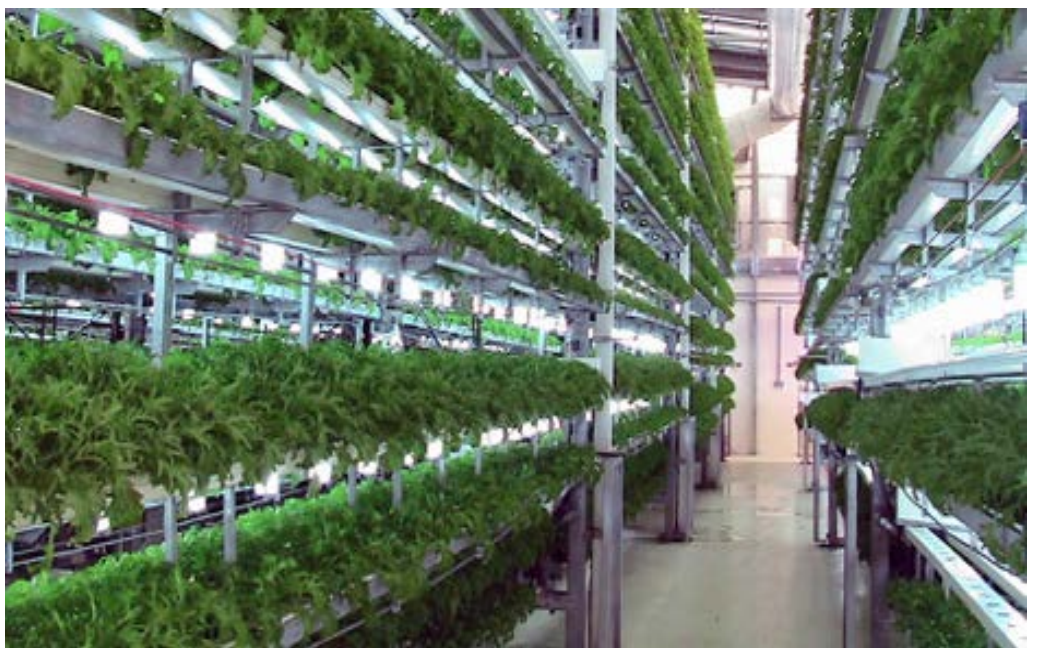

Figure 4. Simulate greenhouse.

using 2.5 G. Cloud server has strong computing power to help the customer make a market decision, and scheduling by using a machine learning algorithm. After computing, the data will both send to the local server and user interface. Local server will send data to actuators, and the user interface will receive data to inform users. Overall those functions and devices, IoT system makes the greenhouse become automated.

\subsection{Sensors}

The five types of sensors are employed in the sensor network. The humidity and temperature sensors, light sensors, and $\mathrm{CO}_{2}$ sensors are in the air; $\mathrm{EC}$ and $\mathrm{pH}$ sensors, and the dissolved oxygen sensors are in the water.

\subsubsection{Humidity and Temperature Sensor}

Humidity and Temperature Sensor: Severe symptoms will occur to plants if the growing temperature and humidity are not within the optimum range. Therefore, it is necessary to have sensors for monitoring the temperature and humidity around the plant. An example of such a sensor is SHT75 (see Figure 5), the digital pin-type relative humidity and temperature sensor produced by Sensation. It provides digital temperature measurements from $-40^{\circ} \mathrm{C}$ to $123.8^{\circ} \mathrm{C}$ with an error range of $\pm 0.3^{\circ} \mathrm{C}$; and humidity measurements from 0 to $100 \% \mathrm{RH}$ (Relative Humidity) with an error range of $\pm 1.8 \% \mathrm{RH}$ [3].

\subsubsection{Light Sensor}

Light exposure creates a profound influence on crop growth in the natural environment. Too high light intensity could burn the leaf; crops cannot the plants will not produce full fruit with too low light intensity. To further illustrate that, by simply installing a photoresistor (see Figure 6) on the SHT75, the light intensity could be measured. When light intensity changes, the resistance of the photoresistor changes and consequently generates an analog signal. The analog signal is transmitted to and processed by the local host. 


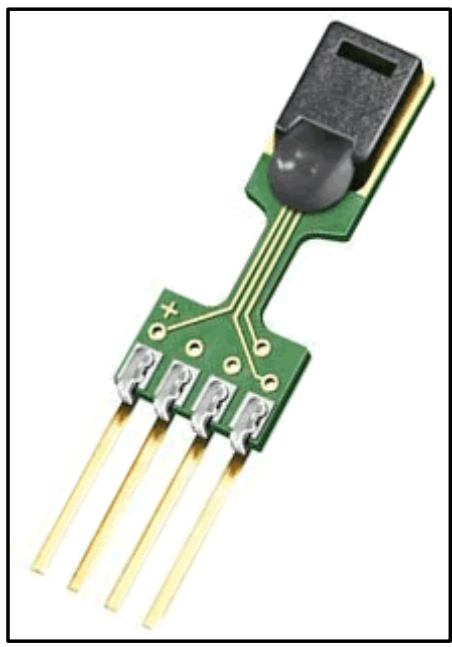

Figure 5. SHT75.

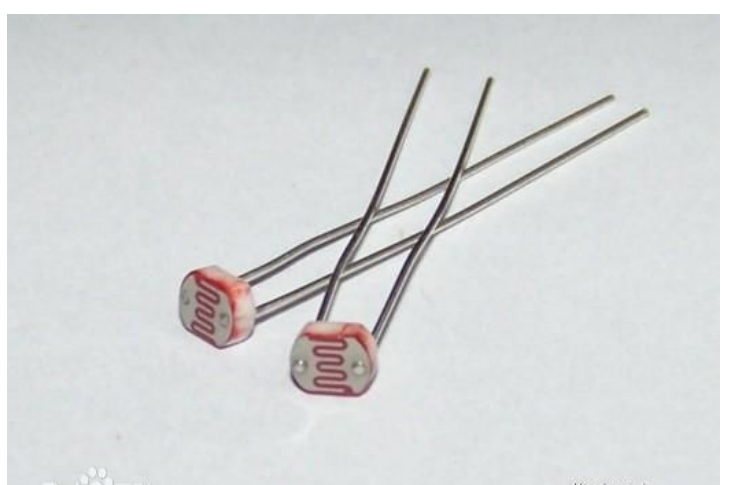

Figure 6. Photoresistor.

\subsubsection{EC and $\mathrm{pH}$ Sensor}

Electrical conductivity (EC) and $\mathrm{pH}$ are two important conditions for hydroponics since each plant have specific conditions to grow. Whether they are higher or lower will lead to death. "EC and $\mathrm{pH}$ probes (the bits that go in the water) typically can't be read directly with a microcontroller. They require an analog circuit to drive them." [4] Cooking-Hacks sensors (see Figure 7) could monitor the $\mathrm{EC}$ and $\mathrm{pH}$ at the same time.

\subsection{4. $\mathrm{CO}_{2}$ Sensor}

Photosynthesis will affect the plant's growth rate, which will stay in a relative low level in the low $\mathrm{CO}_{2}$ concentration, the content of $\mathrm{CO}_{2}$ in the air could control the photosynthesis rate of plants so as to make sure the plant could mature at the time farmer need. The SprintIR Wide Range Carbon Dioxide Sensor (see Figure 8 ) is one of the fastest ( 20 measurements per second), most accurate $\mathrm{CO}_{2}$ sensor on the market [5].

\subsubsection{Dissolved Oxygen Sensor}

Ensuring the root could breathe normally, the water needs enough oxygen. However, too much oxygen will also kill the roots. That is the reason to monitor 


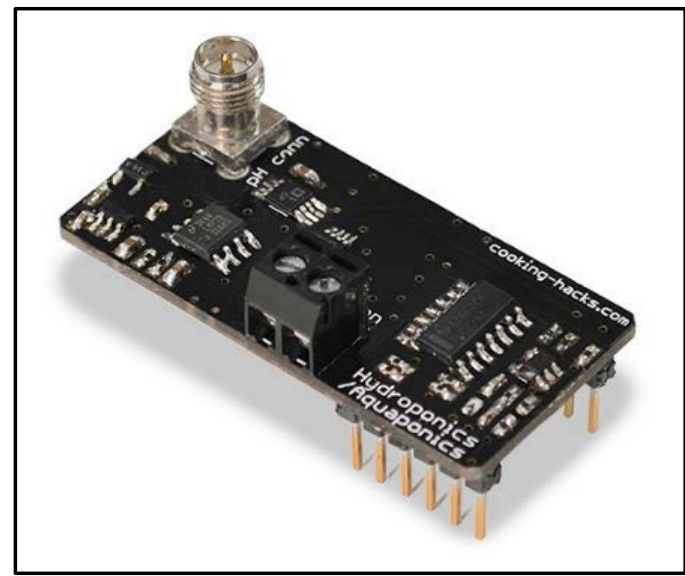

Figure 7. Cooking-hacks sensor [4].

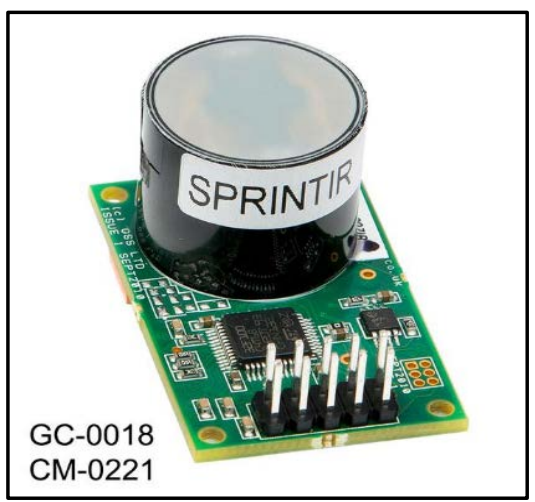

Figure 8. Wide range carbon dioxide sensor [5].

the dissolved oxygen. Through fiber-optic dissolved oxygen sensor (see Figure 9), the concentration of oxygen is easy to be detected [6].

\subsection{Actuators}

There are five actuators that adjust circumstance in order to achieve the ideal atmosphere environment, water condition, and light. For atmosphere, $\mathrm{CO}_{2}$ generator, dehumidifier, and air-conditioner are required. In water, a pump system will adjust the water condition.

\subsection{1. $\mathrm{CO}_{2}$ Generator}

To increase $\mathrm{CO}_{2}$ concentration in the air, we need to use a $\mathrm{CO}_{2}$ generator. For example, $\mathrm{CHP} /$ Micro-CHP (see Figure 10) is one kind of $\mathrm{CO}_{2}$ generator, which burn propane or natural gas to emit $\mathrm{CO}_{2}$ into the atmosphere and generate heat and electricity [7].

\subsubsection{Dehumidifier}

An industrial dehumidifier (see Figure 11) will be used to dehumidification by condensation (cooling the air the water vapor phase to a liquid [8]) and reheat the air. 


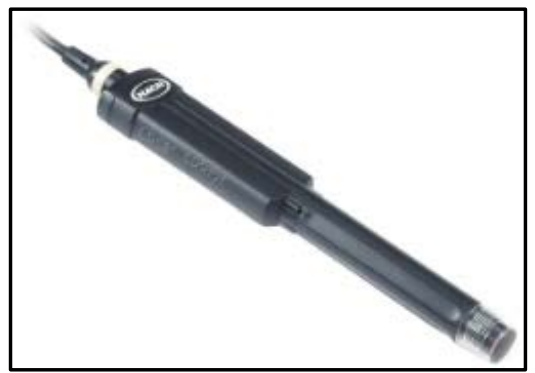

Figure 9. Dissolved oxygen sensor [6].

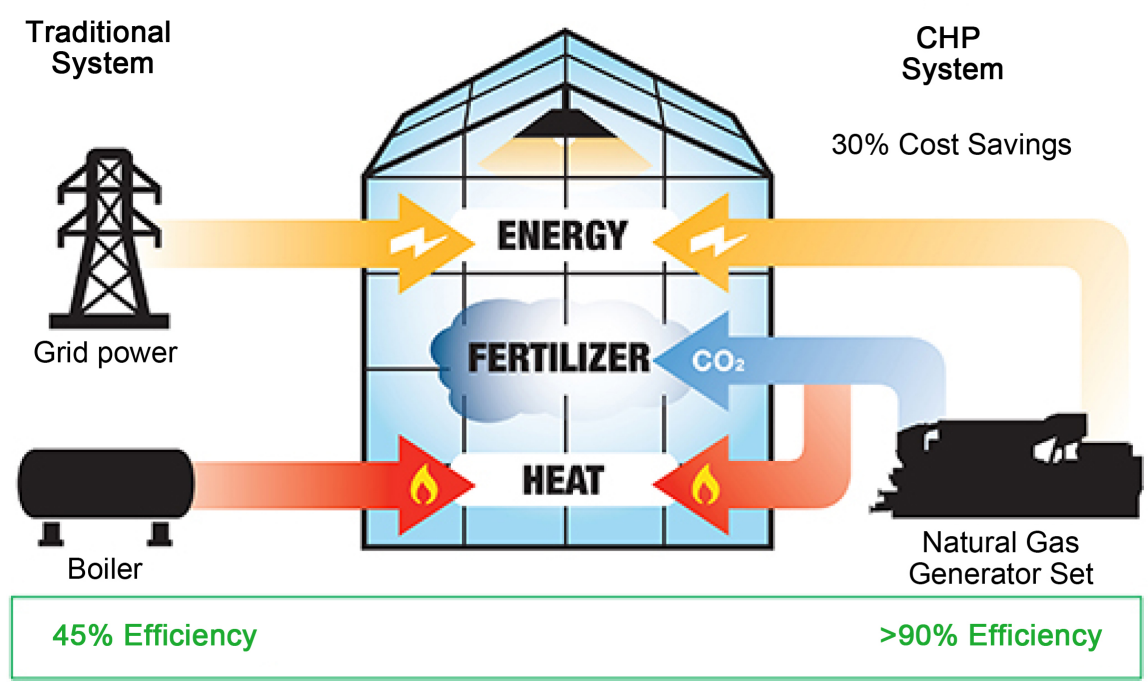

Figure 10. CHP system.

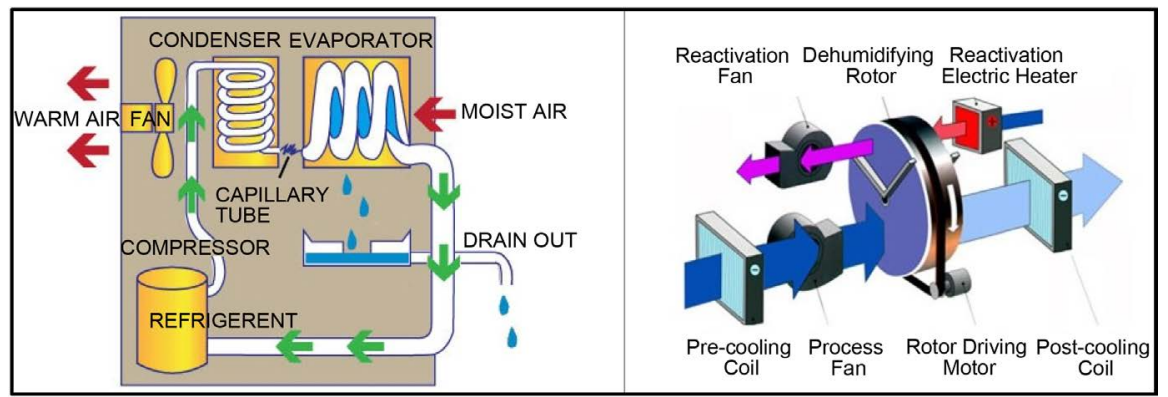

Figure 11. Dehumidifier.

\subsubsection{Air-Conditioner}

Temperature is control by CHP and absorption refrigerator. CHP burn the fuel to heat. And the absorption refrigerator (see Figure 12) will cool the air.

\subsubsection{Pump System}

A pump system contains water pump and air pump. A water pump system, such an auto-dosing system (see Figure 13), can control water condition by pumping the different liquid. For example, adding consistent $\mathrm{pH}$ down or $\mathrm{pH}$ up can adjust $\mathrm{pH}$, and pumping water or nutrient solution can control EC. The air pump can pump air into the water to increase DO. 


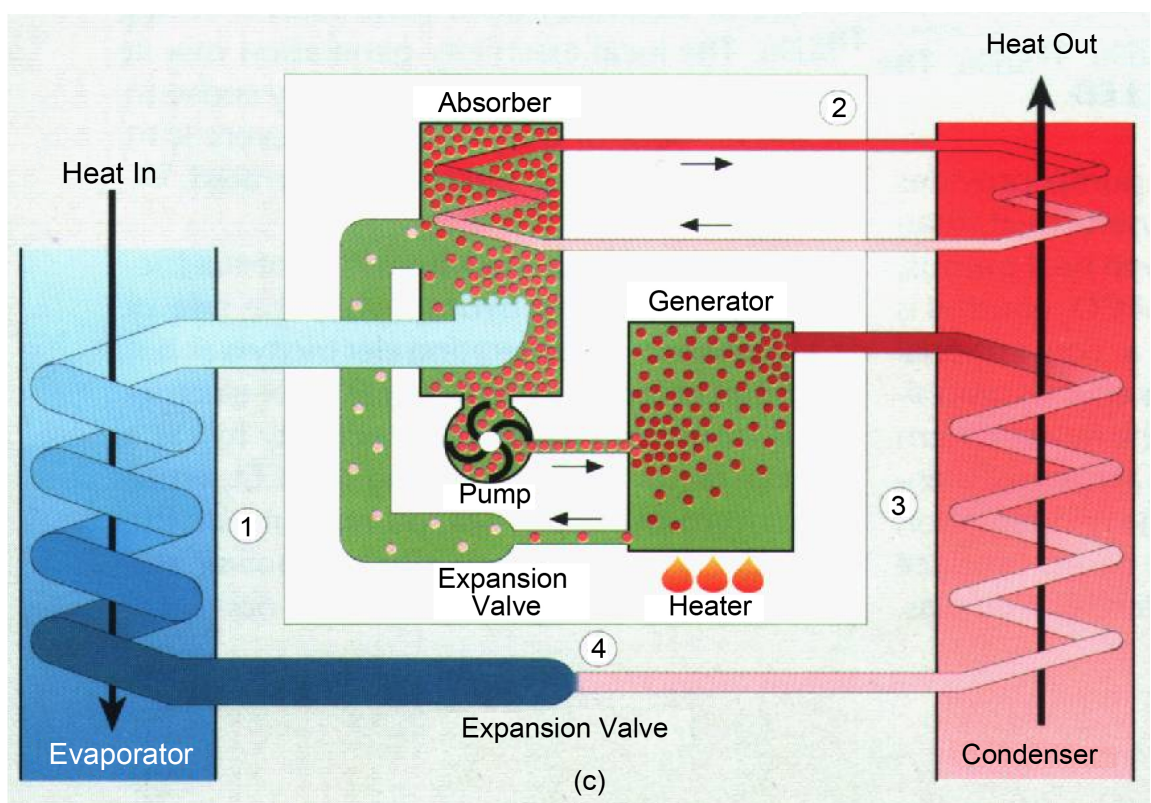

Figure 12. Absorption refrigerator.

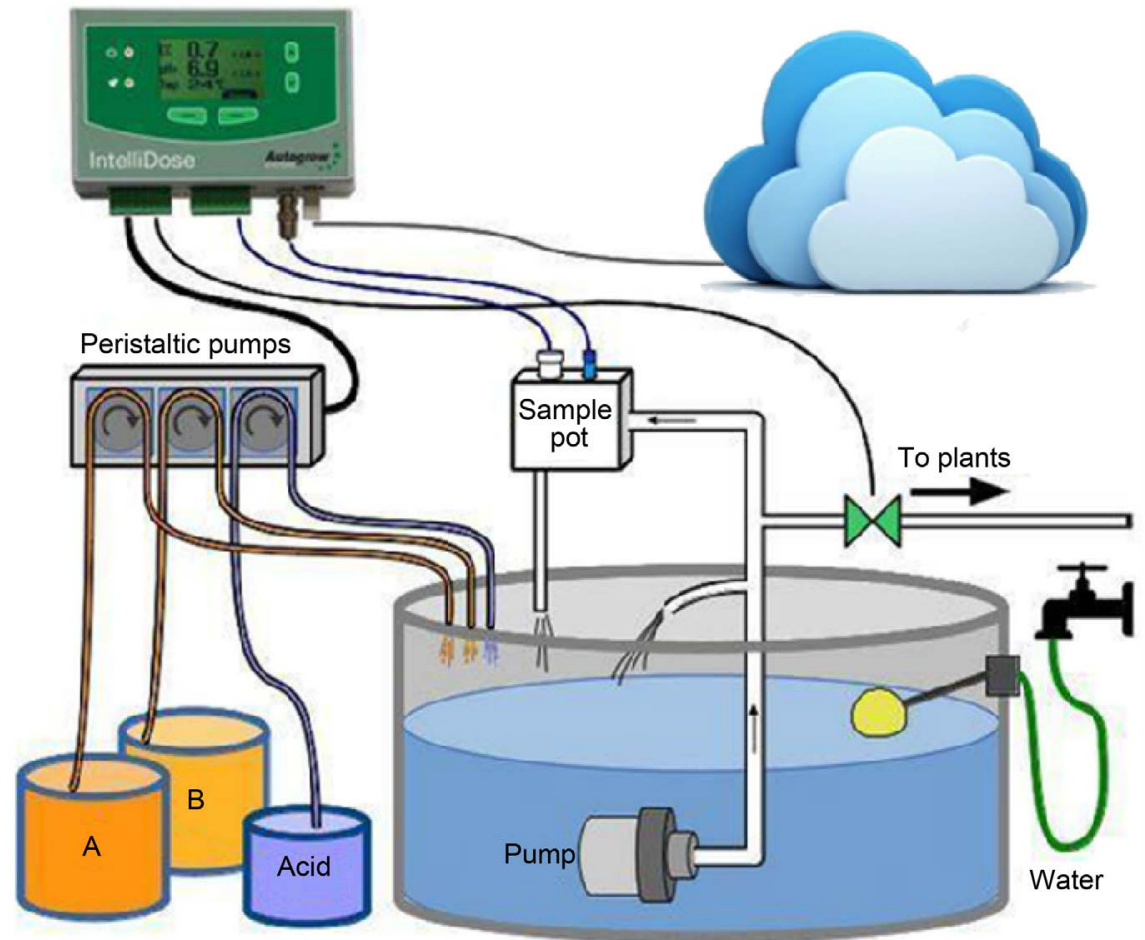

Figure 13. Auto-dosing system [p1].

\subsubsection{Light}

LED light bar is used to enhance light. Different light intensity will control by an open different number of LED light bar (see Figure 14).

\subsection{Communication}

Under the IoT greenhouse environment, numerous sensors, as we introduced in 
the former part and actuators can be utilized for connection throughout the greenhouse, capable of monitoring and detecting the change in the environment. The readings from these sensors can be used in analytics and providing to supervision applications, thus maintaining seamless connectivity between devices and the server become important to the system, especially when we look for precision in operation.

For the network that connects sensors and actuator, we choose to use ZigBee. ZigBee is a protocol that had been developed based on IEEE 802.15.4 which defines the physical and MAC layers. The characteristic that ZigBee support mesh topology with very-low power consumption makes it the most attractive wireless device to use in our project. Since it has multi-hop communication capability, it provides a relatively long range for communication [9]. Figure 15 shows our architecture of mesh network for greenhouse IoT system. A platform embedded with $\mathrm{RF}$ receiver and microcontroller units serves as a local host in the network.

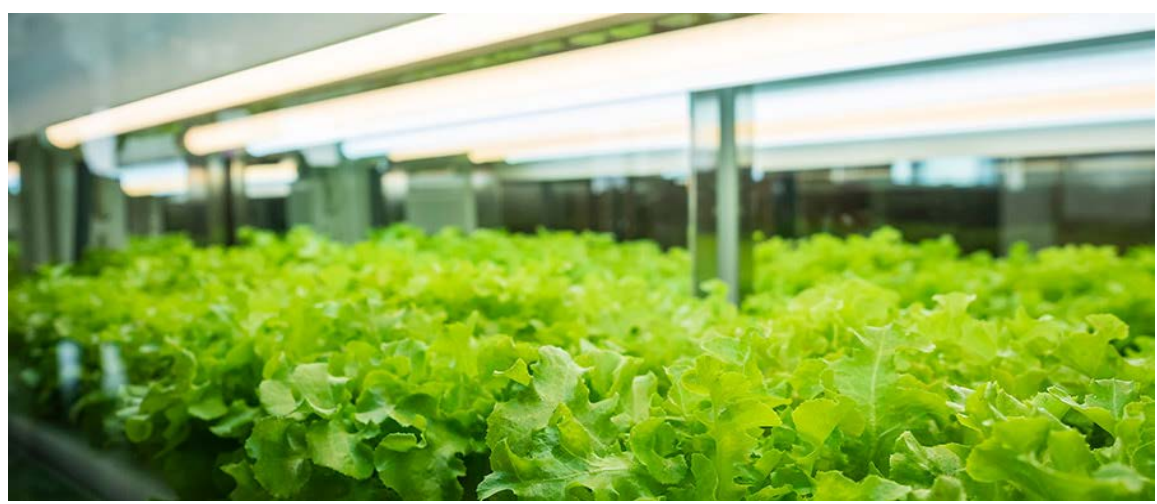

Figure 14. LED light image.

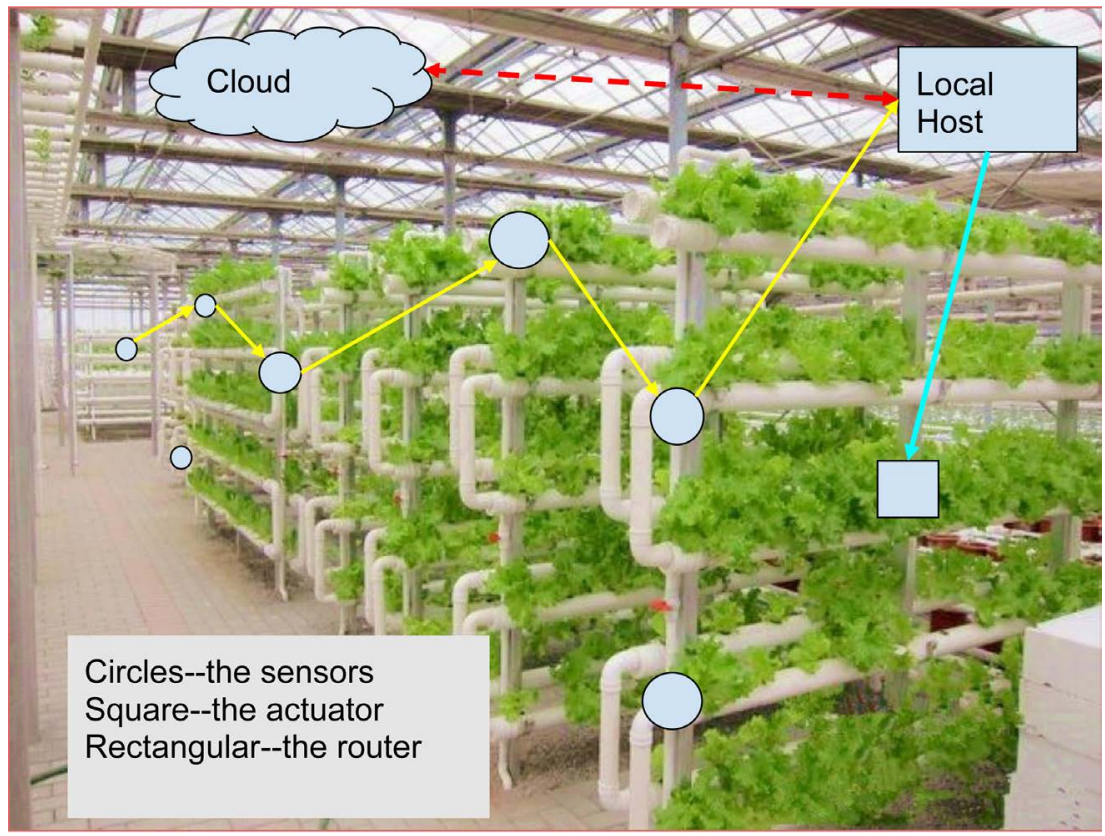

Figure 15. Multi-hopping in network. 
The farther sensors send the message to other sensors, close to the local host, which can receive the data from sensors which can retransmit these messages too much closer sensors and finally to the local host. Then, the analyzed data will be sent to the actuators to stable the environment. The data from each greenhouse will also be forwarded to the cloud server using TCP/IP protocols by the 2.5 G networks, such as GPRS, depends on our need for transmission length.

Since we are talking about a greenhouse, it means the transmission will happen in a relatively short range. There is also another low consumption wireless technology such as Lora, but we don't need to handle the transmission for more than 100 meters or strong interference problems, so ZigBee will be enough for a greenhouse environment. Combining with the mesh network, the data and control messages can be passed from one node to another node through multiple paths, which extends the range of the network and can be competent when we need to manage many devices at the same time, and also ensure the reliability of communication [10].

For the regular wireless network, a lot of energy consumption happened when receiver detecting signals while no call comes in, in another word, idle listening. Now with the LPWAN technology, we can set the base station to stay in low power mode on a condition that there is no need to transmit-receive or control. CPU will only wake up when localhost received commands from the upper layer. Under such circumstance, the ratio of idle time to the total waking up time will be surely shortened and thus prolong the devices' power endurance [11].

The further transmission from the base station to the cloud server will be processed on the $2.5 \mathrm{G}$ network. In order to connect to the cloud servers, we set a two-way satellite connection, which connected to the server which coordinates the sensor patches and provides a relational database service. We would expect to use turnkey base stations since they need to run unattended.

\subsection{Data Storage and Connect Reliability}

Data is collected and arranged in the Database. The data, such as Temperature, humidity, $\mathrm{pH}$ value, and daylight explosion, is sort by different plant or different greenhouse area which is mapping to its Crop Virtual Entity, and prepare for Analytics. Moreover, the server will monitor the link or localhost to make sure the reliability of the connection. If the server does not receive data then it will ask local to retransmit again. If still no response, the server will assume that there must an error on link or host, and send an alert to the user.

\subsection{Analytics}

In order to formulate a forecast model that precisely predicts the growth and crop yield in the greenhouse, we embed crop simulators into the Analytics Layer of the system. According to USDA (United States Department of Agriculture), "crop simulators are computer programs that mimic the growth and development of crops. Data on weather and crop management are processed to predict 
crop yield, maturity date, effectiveness of fertilizers, and other elements of crop production." [12] To be more specific, our system collects data of the environment such as temperature, humidity, $\mathrm{pH}$ value, daylight explosion via the sensor network. Because of the complication of the data processing and forecast making, an appropriate model is needed. On the one hand, artificial neural network (ANN) methodology, designed for dynamic nonlinear systems like growth and development of crops, is an appropriate model and simulation tool [13]. In the recent decades, the ANN model has been used in crop development modeling [14] [15] and crop yield prediction [16] [17] [18] On the other hand, ANN has a better capability in yield prediction than regression and blind guess methods. In this situation, the ANN-6 and ANN-10 are suitable models for prediction [13].

Once the data is collected and transmitted from the service layer, machine learning algorithms such as decision tree learning, artificial neural networks (ANN), and Bayesian networks accommodate the collected data and then formulate simulation models. The machine learning algorithms-along with statistical models, mechanistic models, and functional models [19] - form the forecast model that illustrates the future growth and yield of the plants.

In the market demand, deep neural network for historical data processing, which includes different kinds of crops price and demand of particular crop, is used, so the system can predict the quantity demanded and price of particular types of plants in the upcoming local market. To be specific, because of complicity of the demand and price of crops, we cannot simply utilize a linear regression model. However, these two machine learning algorithms, 1) Recurrent Neural Network (RNN) and 2) Long Short-Term Memory (LSTM), are suitable for our prediction of crops demand, which is nonlinear and influenced by multiple factors [20].

Through the data collection and procession, our IoT system will output a plain and palpable conclusion. For example, our IoT system can help farmers to decide what to plan even if they have no knowledge about market. It can also help farmers to control the whole system with diversification plants even if they have no experience of planting certain type of food. The IoT system makes our greenhouse to be automated.

Eventually, data collected from the greenhouse could further improve the forecast models. This data will be saved in the database and functional data will be sent back to local server.

\subsection{User Interface}

Users at the mobile end are able to access information about the greenhouse via different kinds of user interfaces. A mobile application is discussed below as an example. First of all, the mobile application consists of "Home", "Explore", "Alert", and "My Account". The framework of the application is demonstrated in Figure 16. Users can overview the functions of this app in the menu interface (Figure 17). 


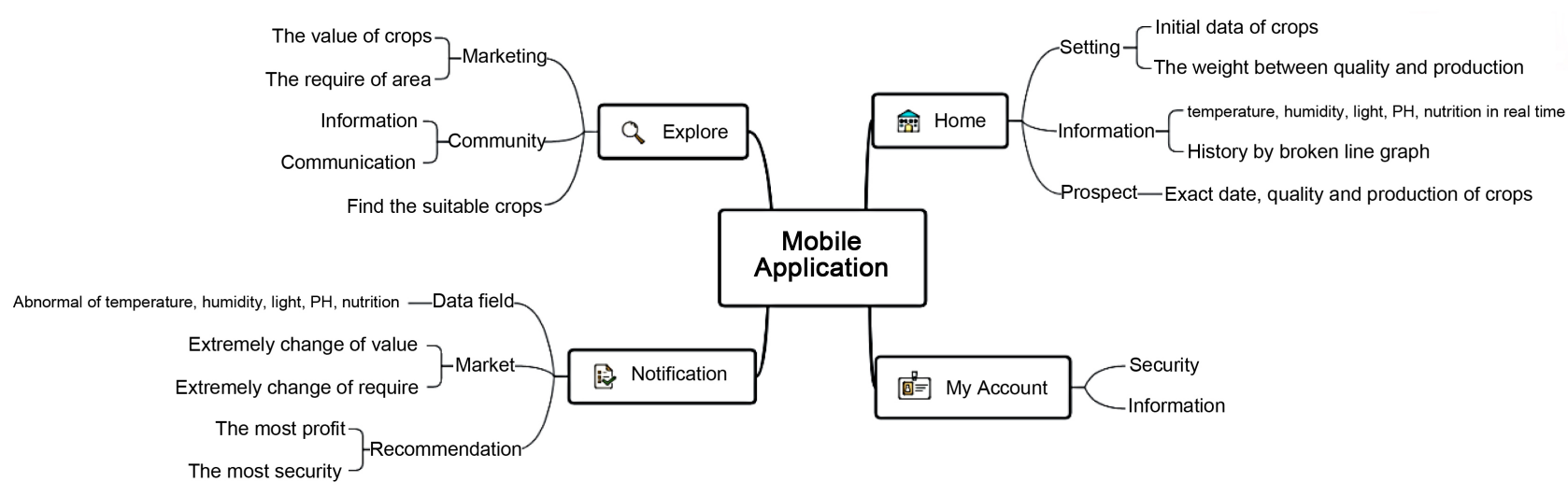

Figure 16. The framework of the application.

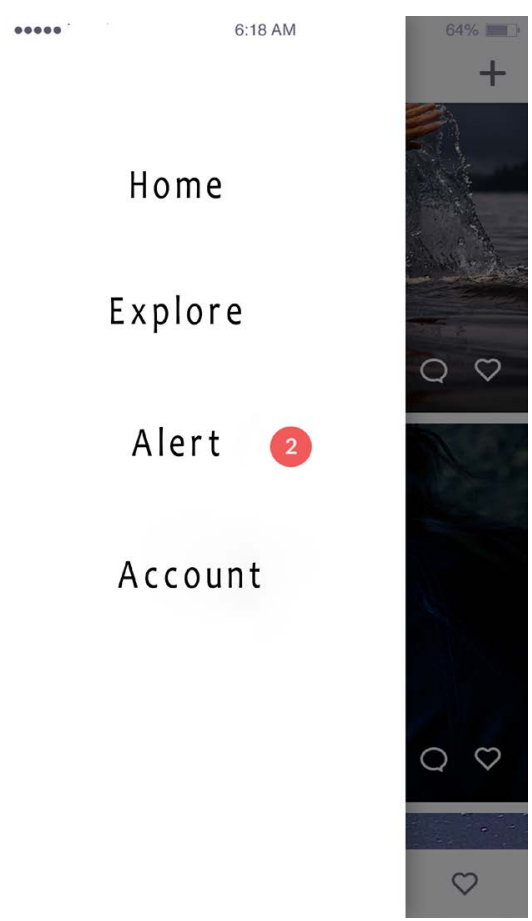

Figure 17. The menu.

"Home": The "Home" page consists of three sections, "Initial Setting" (displays the type and quantity of each plant), "Supervision" (displays temperature, humidity, light, $\mathrm{pH}$, and $\mathrm{CO}_{2}$ measurements) (Figure 18), and "Prospect" (shows harvest date, predicted yield and price of each plant).

"Explore": The "Explore" page has two sections, "Market" and "Trading". The "Market" section provides the users with information about the predicted future prices of different categories of plants and the demanded price at a future date (Figure 19). The "Trading" section features a trading platform that allows users to sell their products.

"Alert": The "Alert" page notifies the users about extreme conditions or critical changes in the market and in the greenhouse. To be more specific, if there are abnormal measurements received by the cloud, if the nutrients to cultivate the 


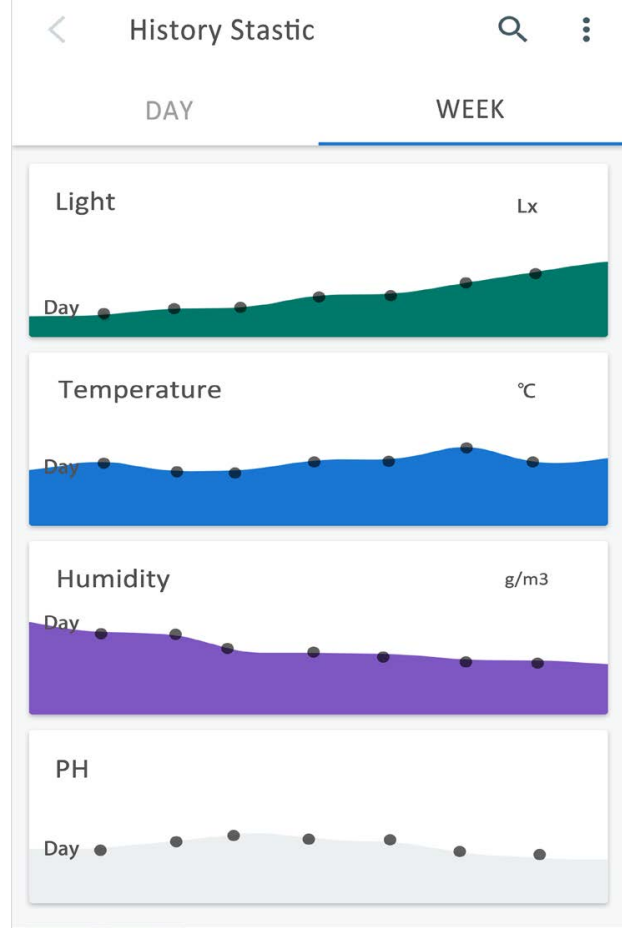

Figure 18. Supervision table.

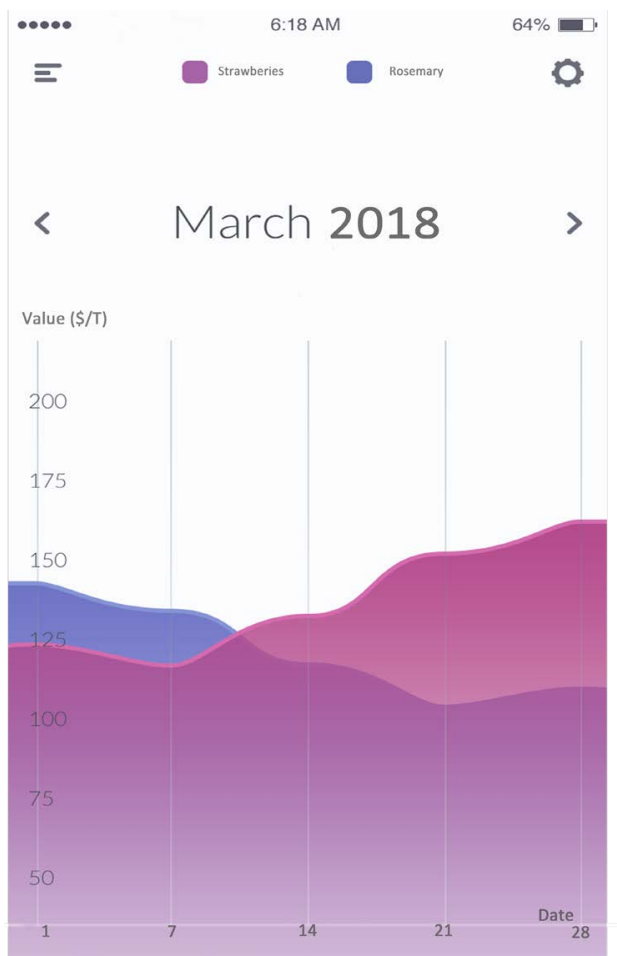

Figure 19. Market table.

plants are out of stock, and if a natural disaster takes place, alerts are immediately shown on this page.

"My Account": The "My Account" page also has two sections, "Settings" and 
"Wallet". The "Setting" section has features such as "Account Security" and other general setting options. The "Wallet" section is the User's digital wallet that shows the balance within the user's account.

\section{Discussions}

\subsection{Evaluation}

While some greenhouses today have installed a monitor system, they still require human labor to adjust the settings or equipment. On the other hand, although an automatic controlling system has been applied to some greenhouses, it lacks the reference standards that maximize the yield and enhance the quality of crops. Instead, our IoT-based hydroponic greenhouse integrated with both the monitor system and the automated controlling system takes advantage of the analytics of massive data from the cloud [21]. With machine learning algorithms like ANN to process the collected data, the cloud generates decision of adjustments in temperature, humidity, $\mathrm{pH}$ value, and light exposure and immediately sends them to the automatic controlling system.

\subsection{Future Outlook}

The amount of arable land has been decreased by one third in the past 40 years, resulting from soil degradation, climate change, and other destructive human activities [22]. Fortunately, the IoT-based hydroponic greenhouse is able to provide access to safe and nutritious food with all humanity, combating the challenging conditions and ensuring food security. Agriculture is no longer an industry that requires massive human labor. The IoT system will fuel a new era of industrialization in agriculture. Conventional risks and damages in agricultural activities could be quantified and eliminated by precise automation. By growing the crops in a greenhouse that perfectly resembles the natural environment, the crops are cultivated organically and safely.

Extending the application scenarios of the IoT system and taking it to the next level, it could be installed in space stations because of the absence of human labor in the IoT greenhouse production. Farming experiments are one of the most popular subjects in space stations. For instance, NASA has initiated the Advanced Plants Habitat (APH), a fully enclosed, closed-loop system with an environmentally controlled growth chamber that will be used to conduct plant bioscience research on the International Space Station [23]. Since APH requires minimal crew involvement, our greenhouse IoT system that automatically stores data and strictly controls the environmental conditions can be installed in APH and ultimately progress space farming.

\section{Conclusion}

Our greenhouse, driven by market demands and based on optimization schedule, is an integration of IoT with agriculture. The automation and high efficiency on monitoring and controlling greenhouse environment are crucial. Such 
a system can be easily installed and maintained, and the manager of this system can access all necessary data collecting from the sensors. We have expressed the benefits of an IoT-based greenhouse to meet the needs of local communities with the best prices and maximized profits.

\section{Conflicts of Interest}

The authors declare no conflicts of interest regarding the publication of this paper.

\section{References}

[1] Internet of Things Global Standards Initiative. https://www.itu.int/en/ITU-T/gsi/iot/Pages/default.aspx

[2] Internet of Things (IoT): A Vision, Architectural Elements, and Future Directions. Future Generation Computer Systems, 29, 1645-1660. https://www.sciencedirect.com/science/article/pii/S0167739X13000241

[3] SHT7x (RH/T) -Digital Humidity Sensor. https://www.sensirion.com/en/environmental-sensors/humidity-sensors/pintype-di gital-humidity-sensors/

[4] Akopyan, V.A. (2016) EC and PH Sensors for Arduino. https://blog.quickbird.uk/ec-and-ph-sensors-for-arduino-98bb70c756b9

[5] SprintIR-Wide Range $100 \% \mathrm{CO}_{2}$ Sensor. https://www.co2meter.com/products/sprintir-100-percent-co2-sensor?variant=2126 93617\&utm_medium =cpc\&utm_source=google\&utm_campaign=Google\%20Shopping\&gclid=Cj0KCQjw45_bBRD_ARIsAJ6wUXQ5zmrIPdC0ujzaztLKjNV2934aP_ S3hE51VejxJiQznZwd2dNHFgsaAkiMEALw_wcB

[6] Intellical ${ }^{\mathrm{TM}}$ LDO101 Laboratory Luminescent/Optical Dissolved Oxygen (DO) Sensor, $1 \mathrm{~m}$ Cable.

https://www.hach.com/intellical-ldo101-laboratoryluminescent-optical-dissolved-o xygen-do-sensor-1-m-cable/product?id=7640489854

[7] Gas-Fueled CHP Delivers Heat, $\mathrm{CO}_{2}$ for Greenhouses. Consulting-Specifying Engineer, 25 July 2014.

https://www.csemag.com/articles/gas-fueled-chp-delivers-heat-co2-for-greenhouses/

[8] Condensation. IUPAC Gold Book. http://goldbook.iupac.org/html/C/C01235.html

[9] Chen, J.Y. and Zhou, X.P. (2006) ZigBee Wireless Communication Technology in Industrial Controls. Radio Engineering of China, 36, 61-64

[10] Pekhteryev, G., Sahinoglu, Z., Orlik, P. and Bhatti, G. (2005) Image Transmission over IEEE 802.15.4 and ZigBee Networks. 2005 IEEE International Symposium on Circuits and Systems, Kobe, Japan, 23-26 May 2005.

[11] Wei, W.D. (2010) The Design of Embedded Wireless Monitoring System Based on ZigBee Technology. Applied Mechanics and Materials, 34-35, 1109-1113. https://doi.org/10.4028/www.scientific.net/AMM.34-35.1109

[12] The United States, Department of Agriculture. (2019) What Are Crop Simulation Models?

https://www.ars.usda.gov/northeast-area/beltsville-md-barc/beltsville-agricultural-r esearch-center/adaptive-cropping-systems-laboratory/docs/what-are-crop-simulati on-models/ 
[13] Dai, X., Huo, Z. and Wang, H. (2011) Simulation for Response of Crop Yield to Soil Moisture and Salinity with Artificial Neural Network. Field Crops Research, 121, 441-449. https://doi.org/10.1016/j.fcr.2011.01.016

[14] Zhang, J.Q., Zhang, L.X., Zhang, M.H. and Watson, C. (2009) Prediction of Soybean Growth and Development Using Artificial Neural Network and Statistical Models. Acta Agronomica Sinica, 35, 341-347. https://doi.org/10.1016/S1875-2780(08)60064-4

[15] Fortin, J.G., Anctil, F., Parent, L. and Bolinder, M.A. (2010) A Neural Network Experiment on the Site-Specific Simulation of Potato Tuber Growth in Eastern Canada. Computers and Electronics in Agriculture, 73, 126-132. https://doi.org/10.1016/j.compag.2010.05.011

[16] Park, S.J., Hwang, C.S. and Vlek, P.L.G. (2005) Comparison of Adaptive Techniques to Predict Crop Yield Response under Varying Soil and Land Management Conditions. Agricultural Systems, 85, 59-81. https://doi.org/10.1016/j.agsy.2004.06.021

[17] Green, T.R., Salas, J.D., Martinez, A. and Erskine, R.H. (2007) Relating Crop Yield to Topographic Attributes Using Spatial Analysis Neural Networks and Regression. Geoderma, 139, 23-37. https://doi.org/10.1016/j.geoderma.2006.12.004

[18] Khazaei, J., Naghavi, M.R., Jahansouz, M.R. and Salimi-Khorshidi, G. (2008) Yield Estimation and Clustering of Chickpea Genotypes Using Soft Computing Techniques. Agronomy Journal, 100, 1077-1087. https://doi.org/10.2134/agronj2006.0244

[19] Basso, B., Cammarano, D. and Carfagna, E. (2019) Review of Crop Yield Forecasting Methods and Early Warning Systems. Department of Geological Sciences Michigan State University, USA.

http://www.fao.org/fileadmin/templates/ess/documents/meetings_and_workshops/ GS_SAC_2013/Improving_methods_for_crops_estimates/Crop_Yield_Forecasting Methods_and_Early_Warning_Systems_Lit_review.pdf

[20] Muthusinghe, M.R.S., et al. (2018) Towards Smart Farming: Accurate Prediction of Paddy Harvest and Rice Demand. 2018 IEEE Region 10 Humanitarian Technology Conference (R10-HTC), Malambe, Sri Lanka, Sri Lanka, 6 December 2018. https://doi.org/10.1109/R10-HTC.2018.8629843

[21] Kodali, R.K., Jain, V. and Karagwal, S. (2016) IoT Based Smart Greenhouse. 2016 IEEE Region 10 Humanitarian Technology Conference (R10-HTC), Agra, India, 21-23 December 2016. https://doi.org/10.1109/R10-HTC.2016.7906846

[22] Soil Erosion: An Agricultural Production Challenge. https://crops.extension.iastate.edu/soil-erosion-agricultural-production-challenge

[23] Advanced Plant Habitat. https://www.nasa.gov/mission_pages/station/research/experiments/explorer/Investi gation.html?\#id=2036 